\title{
Search for fast radio transients using Arecibo drift-scan observations at $1.4 \mathrm{GHz}$
}

\author{
B. B. P. Perera, ${ }^{1 \star}$ A. J. Smith, ${ }^{1}$ S. Vaddi,${ }^{1}$ R. Carballo-Rubio, ${ }^{2}$ A. McGilvray, ${ }^{1}$ A. Venkataraman, ${ }^{1}$ \\ D. Anish Roshi, ${ }^{1}$ P. K. Manoharan, ${ }^{1}$ P. Perillat, ${ }^{1}$ E. Lieb,${ }^{3}$ D. R. Lorimer, ${ }^{4,5}$ M. A. McLaughlin,,${ }^{4,5}$ \\ D. Agarwal, ${ }^{4,5}$ K. Aggarwal ${ }^{4,5}$ and S. M. Ransom ${ }^{6}$ \\ ${ }^{1}$ Arecibo Observatory, University of Central Florida, HC3 Box 53995, Arecibo, PR 00612, USA \\ 2 Florida Space Institute, University of Central Florida, 12354 Research Parkway, Partnership 1, Orlando, FL 32826, USA \\ ${ }^{3}$ Department of Astrophysical \& Planetary Sciences, University of Colorado, Boulder, CO 80309, USA \\ ${ }^{4}$ Department of Physics and Astronomy, West Virginia University, Morgantown, WV 26501, USA \\ ${ }^{5}$ Center for Gravitational Waves and Cosmology, West Virginia University, Chestnut Ridge Research Building, Morgantown, WV 26505, USA \\ ${ }^{6}$ National Radio Astronomy Observatory, 520 Edgemont Rd., Charlottesville, VA 22903, USA
}

Accepted XXX. Received YYY; in original form ZZZ

\begin{abstract}
We conducted a drift-scan observation campaign using the 305-m Arecibo telescope in January and March 2020 when the observatory was temporarily closed during the intense earthquakes and the initial outbreak of the COVID-19 pandemic, respectively. The primary objective of the survey was to search for fast radio transients, including Fast Radio Bursts (FRBs) and Rotating Radio Transients (RRATs). We used the 7-beam ALFA receiver to observe different sections of the sky within the declination region $\sim(10-20)$ deg on 23 nights and collected 160 hours of data in total. We searched our data for single-pulse transients, covering up to a maximum dispersion measure of $11000 \mathrm{pc} \mathrm{cm}^{-3}$ at which the dispersion delay across the entire bandwidth is equal to the $13 \mathrm{~s}$ transit length of our observations. The analysis produced more than 18 million candidates. Machine learning techniques sorted the radio frequency interference and possibly astrophysical candidates, allowing us to visually inspect and confirm the candidate transients. We found no evidence for new astrophysical transients in our data. We also searched for emission from repeated transient signals, but found no evidence for such sources. We detected single pulses from two known pulsars in our observations and their measured flux densities are consistent with the expected values. Based on our observations and sensitivity, we estimated the upper limit for the FRB rate to be $<2.8 \times 10^{5} \mathrm{sky}^{-1}$ day $^{-1}$ above a fluence of $0.16 \mathrm{Jy}$ ms at $1.4 \mathrm{GHz}$, which is consistent with the rates from other telescopes and surveys.
\end{abstract}

Key words: transients: fast radio bursts - stars: neutron - pulsars: general - pulsars: individual: PSR J0625+10, J1627+1419

\section{INTRODUCTION}

FRBs are millisecond-duration energetic radio pulses that are accompanied by large dispersion measures (DMs, where DM is the integral of the column density of free electrons along the line-of-sight from the Earth to the source), placing their origin at cosmological distances. The majority of initial FRBs were discovered with the Parkes radio telescope (Lorimer et al. 2007; Thornton et al. 2013) and since then, more than 600 FRBs have been detected using various other telescopes ${ }^{1,2}$, covering observing centre frequencies below 1.4 GHz (Spitler et al. 2014; Masui et al. 2015; Caleb et al. 2017; Bannister et al. 2017; Shannon et al. 2018; CHIME/FRB Collaboration et al. 2019a; Zhu et al. 2020; Law et al. 2020; Ravi et al. 2019; Connor et al. 2020), and including some follow-up observations at 4-8 GHz (Michilli et al. 2018; Gajjar et al.

\footnotetext{
* E-mail: bhakthiperera@gmail.com

1 https://www. frbcat.org

2 https://www.chime-frb.ca/catalog
}

2018). The DMs of FRBs (currently in the range $\sim 100-3000 \mathrm{pc} \mathrm{cm}^{-3}$; The CHIME/FRB Collaboration et al. 2021) are much larger than the expected DM contributions from the Milky Way in the direction of their lines-of-sight, indicating that the origins must be extragalactic. The FRBs were identified as one-time transient events until the first repeating emission was detected with the Arecibo telescope from FRB121102 (Spitler et al. 2016). Since then, more than 20 FRBs have been identified as repeaters (Luo et al. 2020; CHIME/FRB Collaboration et al. 2019c,b; Kumar et al. 2019). Using follow-up observations with the VLA, FRB121102 was localized to a star-forming region in a dwarf galaxy at a redshift $z$ of 0.193 (Chatterjee et al. 2017), providing further evidence for FRBs that are cosmological in origin. Since then, 17 additional FRBs have been localized to sub-arcsecond precision by radio interferometers, associating them with host galaxies ${ }^{3}$ with known redshifts $z \sim 0.034-0.66$ (e.g., Law et al. 2020; Marcote et al. 2020;

\footnotetext{
${ }^{3}$ http: //frbhosts.org
} 
Macquart et al. 2020; Bannister et al. 2019; Prochaska et al. 2019; Ravi et al. 2019; Chatterjee et al. 2017; Heintz et al. 2020; Piro et al. 2021; Bhandari et al. 2021).

While we have evidence that FRBs are associated with host galaxies, these galaxies have a range of properties, and the FRB origins and formation mechanisms are still a mystery. Determining the prevalence of FRBs across cosmic time could provide significant insight into their formation mechanisms. The Galactic magnetar SGR J1935+2154 emitted FRB-like bursts during its recent active phase (CHIME/FRB Collaboration et al. 2020; Bochenek et al. 2020). These bursts are highly energetic, brighter than any radio bursts seen from Galactic sources, and only a few orders of magnitude lower than the equivalent energy from the faintest FRB (Bailes et al. 2021). This leads to the possibility that at least some FRBs are associated with magnetars (see Lyutikov \& Popov 2020). In order to identify a possible neutron star (NS) origin for FRBs, periodic searches (with periods of order milliseconds to seconds) have been carried out, but these were not successful (Zhang et al. 2018). However, long periodicities of $\sim 16$ days and $\sim 157$ days have been reported for FRB180916B (Chime/Frb Collaboration et al. 2020) and FRB121102 (Rajwade et al. 2020; Cruces et al. 2021), respectively, suggesting that FRB emission mechanisms perhaps align with putative binary systems (Ioka \& Zhang 2020; Lyutikov et al. 2020), slowly spinning neutron stars (Beniamini et al. 2020), and spin precession, including orbit-induced precession (Yang \& Zou 2020), forced precession (Sob'yanin 2020), and free precession models (Levin et al. 2020). Based on the properties of host galaxies, FRBs may also originate through superluminous supernovae and long gamma-ray bursts (for reviews, see Xiao et al. 2021; Zhang 2020a). Moreover, some suggest that FRBs are produced through the magnetospheric activities of magnetars that have been newly formed through binary NS mergers or pre-merger NS-NS interactions (Zhang 2020b; Wang et al. 2020). However, none of these mechanisms are exclusively capable of explaining the origin of FRBs in general.

Recently, Palliyaguru et al. (2021) carried out a search targeting FRBs associated with a sample of 11 gamma-ray bursts that show evidence for the birth of a magnetar. However, this study was unsuccessful in finding FRB-like signals associated with these sources. To uncover the physics of these phenomena, it is essential to increase the known FRB population, to measure a broad range of characteristics such as their duration, emission component structure, spectral properties, polarization, and to also achieve localization in order to measure the properties of the host galaxy. FRBs are detected through single-pulse search algorithms due to their non-periodic, energetic, short-duration, and single-event nature. Many telescopes utilize real-time FRB detection instruments with single-pulse search pipelines to improve the speed and the efficiency of new discoveries, enabling the observations to be done commensally with other projects and ultimately maximizing telescope time for FRB searches and characterization studies (e.g., CHIME/FRB Collaboration et al. 2018; Karastergiou et al. 2015; Foster et al. 2018; Surnis et al. 2019; The CHIME/FRB Collaboration et al. 2021; Niu et al. 2021).

FRBs with high DMs are potentially important as they are generally accompanied by high redshifts and provide a unique opportunity to probe the far reaches of the intergalactic medium. This is crucial for constraining the epochs of hydrogen and helium reionization produced by the ignition of the earliest stars and galaxies (see Keating et al. 2015), leading to a better understanding of the universe in general. The largest DM observed so far is

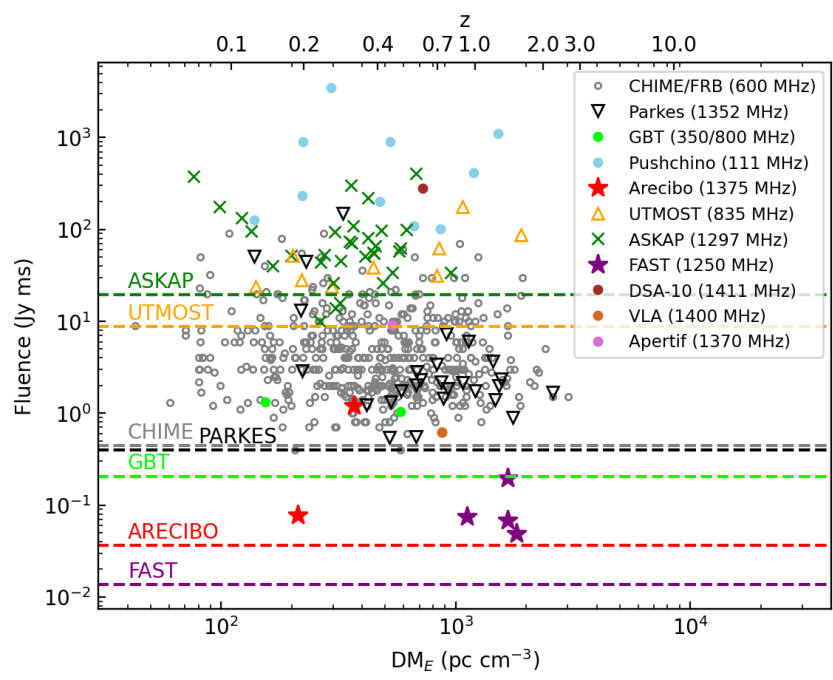

Figure 1. Excess DM and fluence for all known FRBs to-date. The excess DM is obtained after subtracting the expected Galactic contribution based on the electron density model YMW16 (Yao et al. 2017) along the line-ofsight of the source. The upper $\mathrm{x}$-axis represents the redshift and is calculated using the method given in Ioka (2003). More details are given in Section 4. The horizontal dashed lines represent the single-pulse sensitivity for some of the telescopes, assuming a pulse width of $1 \mathrm{~ms}$ and a flat spectral index (see Section 3). The typical center frequencies of these FRB detections and surveys are given in parentheses.

$3038.06 \pm 0.02 \mathrm{pc} \mathrm{cm}^{-3}$ for FRB20180906B ${ }^{4}$, placing its origin at a redshift $>2$ (The CHIME/FRB Collaboration et al. 2021). Some distant FRBs with large DMs exhibit low fluences, indicating the importance of highly sensitive telescopes in detecting those weak sources (Lorimer 2018; Zhang 2018). The highly sensitive 500-m FAST telescope has discovered four FRBs so far, all of which are accompanied by high DMs $\left(>1000 \mathrm{pc} \mathrm{cm}^{-3}\right)$ and low fluences $(<0.2 \mathrm{Jy} \mathrm{ms})-$ e.g., FRB 181017.J0036+11 has a DM of $1845.2 \pm 1 \mathrm{pc} \mathrm{cm}^{-3}$ and a fluence of $0.042 \mathrm{Jy} \mathrm{ms}$, which is the faintest FRB detected so far (Niu et al. 2021; Zhu et al. 2020). Moreover, both FRBs detected by the Arecibo telescope have low fluences -0.08 and $1.2 \mathrm{Jy}$ ms for FRB141113 and FRB121102, respectively (Spitler et al. 2014; Patel et al. 2018) - and are in the lower end of the fluence distribution of known FRBs (see Fig. 1). In general, detecting FRBs with high redshift and low fluence is challenging due to sensitivity limitations of telescopes. Hence, large aperture instruments such as the FAST and Arecibo telescopes are of paramount importance for detecting such weak sources (see Zhang 2018). However, the small field-of-view of such large telescope degrades the speed of the surveys.

RRATs are rotating neutron stars that emit sporadic emission that can be discovered only through single-pulse search algorithms (McLaughlin et al. 2006). They have much more similar emission properties to normal pulsars than FRBs. Unlike FRBs, RRATs are located within our Galaxy ${ }^{5}$. Utilizing a large DM range in singlepulse search algorithms covering the Galactic DMs, we can detect both of these fast transient sources, in addition to normal or giant pulse emitting pulsars, and this is a standard approach in many surveys (see Patel et al. 2018; Parent et al. 2020, for details).

In this work, we carried out a drift-scan observation campaign at

\footnotetext{
4 https://www.chime-frb.ca/catalog

5 http://astro.phys.wvu.edu/rratalog
} 


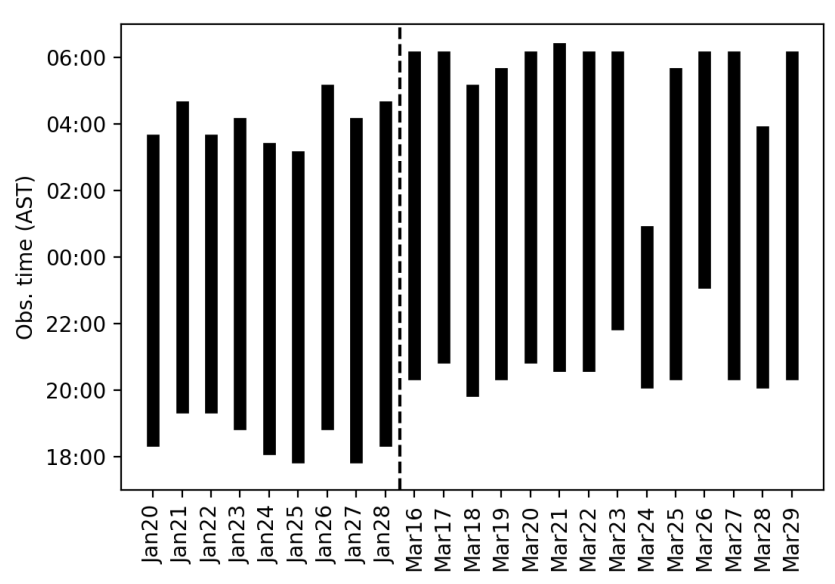

Figure 2. The observation program of the drift-scan campaign. We observed 9 and 14 days consecutively in January and March, respectively. Each day, we started the observation between 18:00-21:00 AST, except on March 26 where we started at 23:15 AST due to technical difficulties. We observed for 8-10 hours each day except only 4.5 hours on March 24 due to data storage issues.

the Arecibo Observatory to search for fast radio transients, including FRBs and RRATs. The paper is organized as follows: we describe our observations, data preparation, and the system performance in Section 2. The sensitivity of our data to single pulses is estimated in Section 3 and we discuss our search pipeline in Section 4. The results of our single-pulse search are presented in Section 5, and the detection of known pulsars in our data and their flux densities are described in Section 6. Finally in Section 7, we discuss results and limits on FRB rates based on our observations.

\section{OBSERVATIONS}

A series of strong earthquakes struck Puerto Rico starting on 28 December 2019, including a magnitude of 6.0 catastrophic earthquake ${ }^{6}$ on 6 January 2020 in the southwest of the island. Following these events, the Arecibo Observatory was temporarily closed for several weeks in January 2020 for safety inspections of the facility. Shortly thereafter, the COVID-19 pandemic also forced a temporary closure of the observatory site during March 2020. During both of these periods, we were able to coordinate and conduct drift-scan observations with the 305-m Arecibo telescope with minimum operational support by recording the data continuously as the sky drifted across the telescope beam at the sidereal rate. We observed everyday between 20-28 January and 16-29 March, resulting in 23 days in total. Each day, we started observations around 20:00 AST (Atlantic Standard Time) and continued taking data for about 8-10 hours. The observation program and the time duration on each day are presented in Fig. 2. We further note that this campaign was conducted during the downtime of the telescope until its normal operations resumed. Therefore, we could not collect more data after this period.

The primary goal of our campaign was to search for new transients in the Arecibo sky. The transient searches typically require high time and frequency resolution. FRBs were mainly detected at $\sim 1.4 \mathrm{GHz}$ frequencies until the recent CHIME telescope detections at $\sim 600 \mathrm{MHz}^{7}$ (The CHIME/FRB Collaboration et al. 2021).

\footnotetext{
6 https://www .usgs.gov/news/magnitude-64-earthquake-puerto-ric

7 https://www. chime-frb.ca/catalog
}

We carried out our observations using the Arecibo L-band Feed Array $^{8}$ (ALFA) receiver with a centre frequency of $1375 \mathrm{MHz}$ and a bandwidth of $322 \mathrm{MHz}$ with 960 frequency channels, resulting in a spectral channel size of $0.335 \mathrm{MHz}$. The two polarization channels were summed together, and we recorded the total intensity data using the Mock spectrometers at a sampling rate of $65 \mu \mathrm{s}$. We note that this setup is very similar to the regular observing configuration of the PALFA survey ${ }^{9}$, which has discovered several fast transients ${ }^{10}$, including both Arecibo-discovered FRBs and also 20 RRATs (Spitler et al. 2014; Patel et al. 2018; Deneva et al. 2009; Parent et al. 2021).

The field-of-view (FoV) is important in regular searching campaigns as it can enhance the instantaneous sky coverage and thus, the survey speed. The ALFA receiver was a seven-beam feed-array which observed seven pixels on the sky simultaneously. Each beam had a full-width-half-maximum (FWHM) power of approximately $3^{\prime} .35$, corresponding to an instantaneous FoV of $0.022 \mathrm{deg}^{2}$ across the 7 pixels (see Cordes et al. 2006; Spitler et al. 2014). With this FWHM beamwidth, the sky transit time between the half power points of the beam was approximately $13 \mathrm{~s}$. Therefore, the ALFA receiver in drift-scan mode provided rapid sky coverage.

With the limited operational support at the observatory in January, we were not able to move the receiver in azimuth and zenith angles. Rather we kept it at the same position (with the exception of January 28), resulting in observing at the same zenith angle. During the March observations, we were able to change the receiver position in azimuth by one degree each day in order to scan different parts of the sky. In addition, the receiver was positioned at a parallactic angle offset of $19^{\circ}$ in all our observations to scan the sky without leaving gaps between the beams (see Fig 3 top panel) and then unchanged during data-taking. Fig. 3 bottom panel shows the observed sections of the sky during the two observing sequences. Several known pulsars and FRBs happened to be within the ALFA beams, and they are marked in the figure. We discuss the detectability of these sources in Section 6.

\subsection{Data preparation}

The data were recorded in PSRfits format ${ }^{11}$ as 16-bit integers, and the lower and upper half of the frequency bands were recorded separately as two individual files. Since our single-pulse searching software supports only 8-bit integers, we compressed the original 16-bit data into 8-bit using psrfits2psrfits ${ }^{12}$ and then merged the two frequency bands using combine_mocks ${ }^{13}$ to produce the full bandwidth of $322 \mathrm{MHz}$. As required by our single-pulse search pipeline, the data were then converted into the filterbank format using digifil ${ }^{14}$ (van Straten \& Bailes 2011). All these tools are commonly used in pulsar data preparation, handling, and processing.

We investigated the radio frequency interference (RFI) environment across the frequency band. By inspecting the dynamic spectra of the data (see Fig. 4 for example), we identified persistent RFI in a large number of frequency channels between 1250 and $1300 \mathrm{MHz}$. In addition, the level of the RFI below $1250 \mathrm{MHz}$ was dynamic and varied over our observation time. Thus, we decided to ignore and excise

\footnotetext{
8 http://www.naic.edu/alfa/

9 http://www2 naic.edu/alfa/pulsar

10 http://www .naic. edu/ palfa/newpulsars

11 https://www.atnf.csiro.au/research/pulsar/psrfits_definition/Psrfit

12 https://github.com/juliadeneva/psrfits2psrfits

$d^{3}$ https://github.com/demorest/psrfits_utils

14 http://dspsr. sourceforge.net/index.shtml
} 

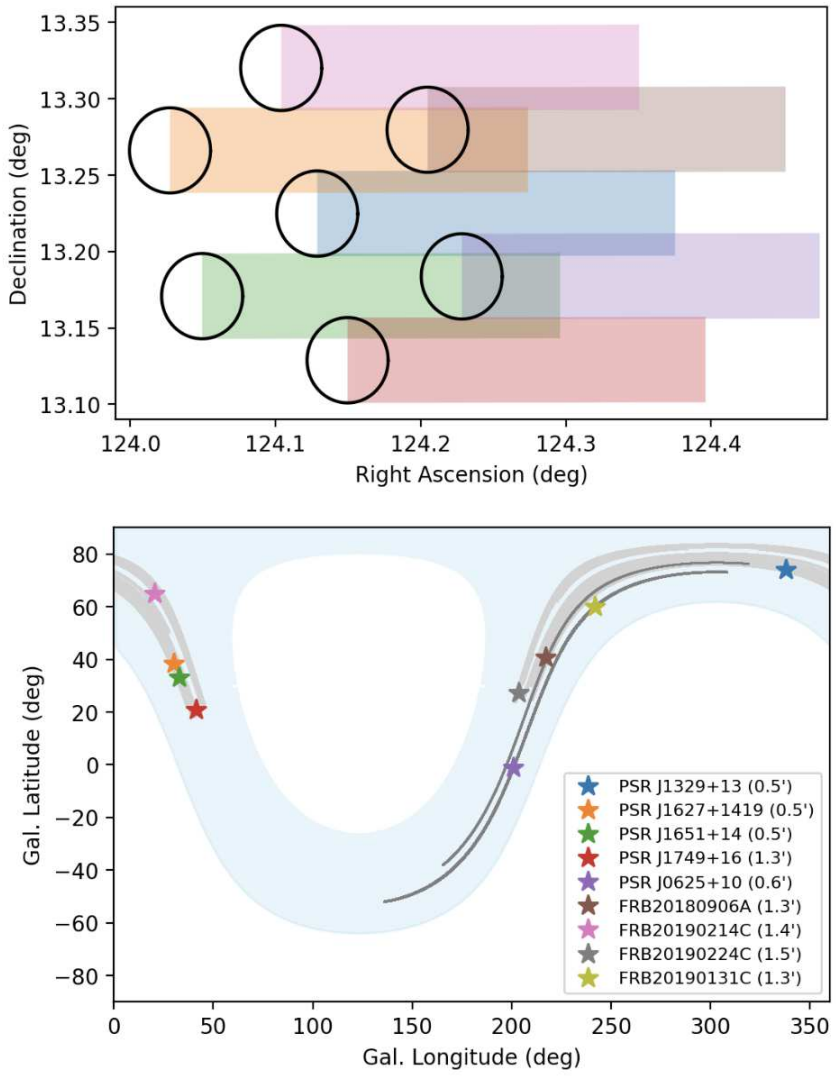

Figure 3. Top: The circles represent the position and the FWHM power of the ALFA receiver beams with respect to the sky at the beginning of the 16 March observation. The color stripes represent the first $60 \mathrm{~s}$ of the observation, showing no gaps across the sky between the beams during the scan. Bottom: The sky regions covered during our drift-scan observations displayed in Galactic coordinates. The blue shaded area represents the portion of the sky visible to the Arecibo telescope within its declination range from $-1^{\circ}$ and $+37.5^{\circ}$. The coverage of January and March observations are marked in dark gray and light gray, respectively. The known pulsars and FRBs within the ALFA beams are marked in different colors, and the offsets from the centre of the closest observed beam are given in parentheses in units of arc-minutes (see Section 6 for details of the detection of these sources).

the frequency channels below $1300 \mathrm{MHz}$ across all our observations. We also identified strong RFI around 1330 and $1350 \mathrm{MHz}$ and excised the relevant channels. The RFI removal reduced the usable bandwidth by roughly one-third, resulting in an effective bandwidth of approximately $215 \mathrm{MHz}$.

\subsection{System gain and stability}

We detected radio continuum emission from many background sources during their transit across the ALFA beams. Based on their sky locations, we confirm that these are identified radio point sources (i.e., source size is smaller than our beam size) reported in the NVSS survey catalog 15 (Condon et al. 1998). These compact continuum sources were detected in almost all beams daily in our observations. Fig. 5 shows the detection of NVSS 145131+134324 on March 17 in the central beam (Beam0) of the receiver. We used these detected

15 https://wWW.cv.nrao.edu/nvss

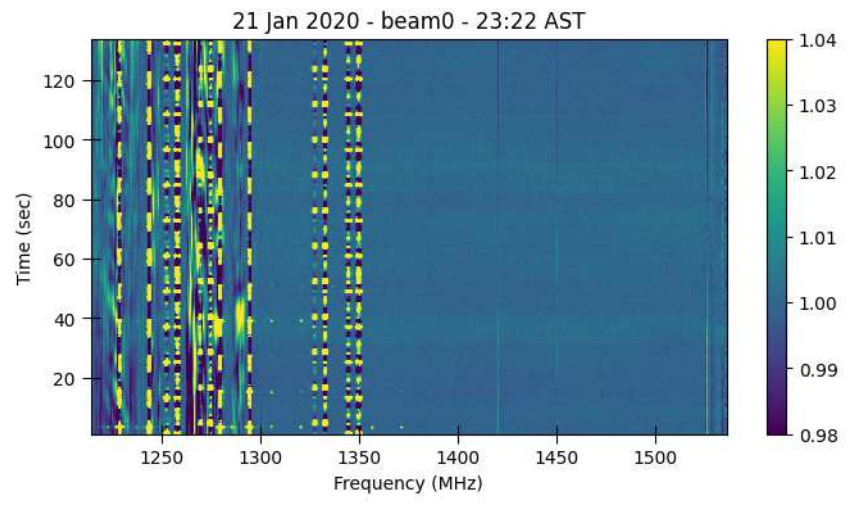

Figure 4. The dynamic spectrum of approximately $130 \mathrm{~s}$ of data obtained on 21 January 2020 at 23:22 AST. A high level of RFI can be seen below $1300 \mathrm{MHz}$ in general and also around 1330 and $1350 \mathrm{MHz}$. We excised the spectral channels corresponding to these frequency ranges in the data processing to mitigate RFI.

sources along with their flux densities reported in the NVSS catalog to estimate the gain of the telescope and the stability of the system throughout our observations. The details of the gain estimation method are described in Appendix A. We estimated the gain for all beams (except Beam5) for most observing sessions. We ignored Beam5 in the gain calculation as one of its polarization channels was unstable and poorly behaved. However, we note that Beam5 was included in the single-pulse search analysis described in Section 4. Our system performance analysis determined that the overall system was stable throughout the campaign, while showing a slight difference in gain between the January and March observing sessions. The average gain of the central beam, Beam0, was estimated to be $8.2 \pm 0.5 \mathrm{~K} \mathrm{Jy}^{-1}$ and that of the other beams was $7.1 \pm 0.8 \mathrm{~K} \mathrm{Jy}^{-1}$ in March observations, which is consistent with the typical system performance of the ALFA receiver ${ }^{16}$. In January, the average gain of Beam 0 and the other beams were $7.5 \pm 0.3$ and $5.6 \pm 0.8 \mathrm{~K} \mathrm{Jy}^{-1}$, respectively. We attribute the lower gain to the inactivity of the tie-down cables of the telescope throughout the January observations, which resulted in defocusing compared to the optimal telescope setup. However, we note that even during this period the system sensitivity was still very high compared to other telescopes in the world, with the exception of the 500-m FAST telescope.

\section{SENSITIVITY TO SINGLE PULSES}

From radiometer noise considerations, the peak flux density of a single pulse,

$S_{i}=\frac{\beta\left(T_{s y s}+T_{s k y}\right)(S / N)_{b}}{G W_{i}} \sqrt{\frac{W_{b}}{n_{p} \Delta f}}$,

where $T_{\text {sys }}$ is the system temperature at the observing frequency, $T_{\text {sky }}$ is the sky temperature in the direction of the telescope pointing, $\beta$ is the factor of sensitivity loss due to digitization, $(S / N)_{\mathrm{b}}$ is the signalto-noise ratio of the broadened pulse, $G$ is the telescope gain, $\Delta f$ is the observing bandwidth, $n_{p}$ is the number of summed polarization channels, $W_{i}$ is the intrinsic pulse width, and $W_{b}$ is the broadened pulse width (see Cordes \& McLaughlin 2003; Patel et al. 2018). The 


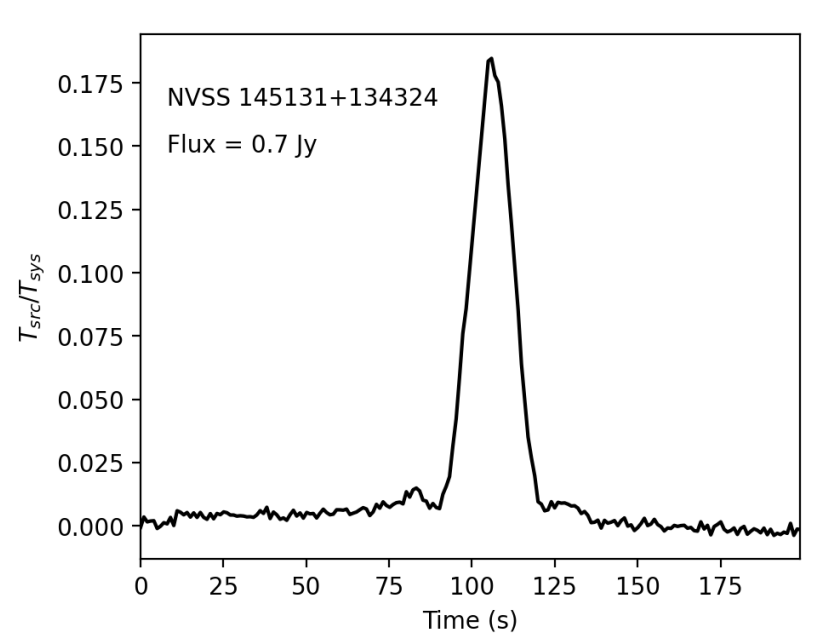

Figure 5. The detection of continuum background source NVSS $145131+134324$ on March 17 in the central beam. The y-axis is scaled as the ratio of the source temperature and the system temperature. The source has a known flux density of $692 \mathrm{mJy}$ at $1.4 \mathrm{GHz}$ and was used to estimate the gain of the telescope as described in Section 2.2.

pulse can be broadened due to various reasons such as dispersion smearing within the frequency channel, scattering, etc. Assuming a telescope gain of $8 \mathrm{~K} \mathrm{Jy}^{-1}$ (see Section 2.2) and typical values for the system $T_{\text {sys }}+T_{\text {sky }} \approx 30 \mathrm{~K}, \Delta f=215 \mathrm{MHz}, n_{p}=2$, and $\beta=1.1$ (Patel et al. 2018), we can rewrite the above expression as $S_{i}=0.006(S / N) / \sqrt{W}$ Jy for pulse width $W_{i}=W_{b}=W$ in units of milliseconds.

The above expression gives a theoretical estimate of the singlepulse sensitivity assuming Gaussian noise in the data. However, the sensitivity in real data can deviate significantly from this formalism due primarily to the presence of RFI and non-Gaussian noise. Based on simulations using PALFA data, Patel et al. (2018) found that the sensitivity of their pipeline to single pulses was degraded by many factors depending on the dispersion measure and the pulse width of the detection. They determined a degradation factor of approximately 1.5 for a pulse with a DM of $1000 \mathrm{pc} \mathrm{cm}^{-3}$ and a width of $5 \mathrm{~ms}$. Since our observation configuration is similar to that of PALFA, we assume the same degradation factor in our study as a conservative value. Assuming a $S / N=8$ and $W=5 \mathrm{~ms}$, the single-pulse sensitivity in our data is then estimated to be $0.032 \mathrm{Jy}$, leading to a fluence $\left(\equiv S_{i} W\right)$ of approximately $0.16 \mathrm{Jy} \mathrm{ms}$. This indicates that the high sensitivity of our data is capable of detecting low fluence FRBs as well as weak single pulses from RRATs.

Following the above method, we estimated the single-pulse sensitivity for different telescopes, assuming a pulse width of $1 \mathrm{~ms}$ (see Fig. 1). We note that the telescopes operate at different frequencies in general. Therefore, we assumed a flat spectral index in the calculation (see Section 7.5 in The CHIME/FRB Collaboration et al. 2021, for discussion). This estimation shows that the Arecibo telescope was capable of detecting all the known FRBs to-date and its sensitivity was second only to the 500-m FAST telescope.

\section{SINGLE-PULSE SEARCH}

Since we are interested in searching for fast transients, we performed a standard single-pulse search analysis. We first determined the DM range to search for according to our observation configuration. The dispersion smearing across the full bandwidth of our data is estimated to be $1.06 \times \mathrm{DM} \mathrm{ms}$, where $\mathrm{DM}$ is in $\mathrm{pc} \mathrm{cm}^{-3}$ (using Equation 4.7 in Lorimer \& Kramer 2012). Therefore, the maximum DM that can be searched for in our data is $\sim 11000 \mathrm{pc} \mathrm{cm}^{-3}$ in order to ensure that the entire dispersed signal covers the bandwidth within the sky transit time of $\sim 13 \mathrm{~s}$, optimizing the $\mathrm{S} / \mathrm{N}$. The signal would only partially cover the bandwidth for DMs greater than this value thus degrading the S/N. According to the relationship between the DM due to intergalactic plasma and the redshift (using Equation 2 in Ioka 2003), this particular DM limit is equivalent to a redshift of $\sim 14.5$, for $\Omega_{m}=0.3$ and $\Omega_{\Lambda}=0.7$ (see Choudhury \& Padmanabhan 2005; Komatsu et al. 2009).

In keeping with standard procedure of transient searches, we first dedispersed the data of each beam with trial DMs in the range of $1-11000 \mathrm{pc} \mathrm{cm}^{-3}$, excluding obvious local RFI with zero DM. The dedispersed data were then averaged in frequency to generate a time series and then searched for single pulses. In order to enhance the $\mathrm{S} / \mathrm{N}$ of single pulses, the time series was convolved with a series of box-car filters with various widths between 1 and 4096 samples (i.e. between $\sim 0.0655-270 \mathrm{~ms}$ in time according to our $\sim 65.5 \mu \mathrm{s}$ sampling time) in power-of-two increments. The information of the selected single pulses (e.g. time stamp, DM, box-car width) above a $\mathrm{S} / \mathrm{N}$ threshold of 6 were saved for further evaluation. All the above steps were completed using the single-pulse search software package HEIMDALL ${ }^{17}$, which processes the data in parallel using GPUs to speed up the incoherent dedispersion (Barsdell et al. 2012). We also note that background continuum sources mentioned in Section 2.2 were removed by subtracting running averages of two seconds from the time series before they were searched for single pulses.

Single-pulse searches in general produce large number of candidates and therefore, it is not feasible to inspect all of them visually. For example, the HEIMDALL package detected several thousand candidates for a given beam for each hour of our data, resulting in more than 80000 candidates for all seven beams per day. We also note that these candidates include a high rate of false positives due to Gaussian noise and RFI in the data. Therefore, machine learning techniques and algorithms have been introduced in single-pulse and pulsar searches to classify the candidates (e.g., RFI/non-astrophysical, FRB, and pulsar candidates). For instance, the deep learning methods have been used in pulsar search campaigns and improved the efficiency of searching procedures significantly (see Zhu et al. 2014; Devine et al. 2016; Guo et al. 2017; Bethapudi \& Desai 2018). Machine learning techniques have also been used in FRB searches (Wagstaff et al. 2016; Foster et al. 2018), including convolution neural network classification methods (Zhang et al. 2018; Connor \& van Leeuwen 2018). After sorting the candidates using classifier models, the high-probability ones can then be visually inspected in order to determine whether they are real astrophysical signals.

We fed all the candidates through the FRB search software FETCH $^{18}$, which uses deep neural networks for classification of FRBs, RRATs, and RFI (Agarwal et al. 2020b; Agarwal \& Aggarwal 2020). FETCH uses single-pulse candidate information from HEIMDALL as described above and produces frequency-time, DM-time images as well as the frequency averaged time series. The convolutional neural networks are applied on these images in the classification process. The software currently includes 11 deep learning models, which were trained using known and simulated FRB, pulsar, and real RFI signals. Agarwal et al. (2020b) reported that these models have an accuracy above $99.5 \%$ on their test data set which consists of real

\footnotetext{
17 https://sourceforge.net/projects/heimdall-astro

18 https://github.com/devanshkv/fetch
} 
RFI and pulsar candidates. We note that FETCH runs on GPUs, which accelerates the image creation of candidates and the classification significantly by processing several candidates in parallel. In this process, each candidate was assigned a probability from 11 separate classifier models. We first sorted and inspected the candidates based on their probabilities produced by the classifier Model $a$, which has the highest prediction accuracy of $99.8 \%$ (see Agarwal et al. 2020b). We also summed up the probabilities from all 11 classification models to obtain the score (out of 11) for each candidate and proceeded to sort and visually inspect each one.

We note that both FETCH and HEIMDALL have been used in many searches and have also been integrated into transient search pipelines (e.g. Agarwal et al. 2020a; Law et al. 2020). As an independent test, we applied our single-pulse search pipeline on the raw data for RRATs J1908+13 and J1924+10 that were recently discovered by the PALFA collaboration ${ }^{19}$. Our pipeline re-detected these sources successfully with high maximum single-pulse $\mathrm{S} / \mathrm{N}$ values of 34 and 14 for J1908+13 and J1924+10, respectively. These S/N ratios are slightly higher than the discovery-reported $\mathrm{S} / \mathrm{N}$ values based on the PRESTObased (single_pulse_search.py) pipeline (Ransom et al. 2002; Keith et al. 2011; Ransom 2011). We note that it is known that the two methods can report slightly different $\mathrm{S} / \mathrm{N}$ ratios based on their data processing frameworks; see Aggarwal et al. (2021) and Gupta et al. (2021) for a detailed discussion.

\section{SINGLE-PULSE CANDIDATE RESULTS}

The data processing produced more than $1.8 \times 10^{6}$ single-pulse candidates with $\mathrm{S} / \mathrm{N}>6$, but we reiterate that the false positive rate of these candidates is very high. We first sorted the candidates based on the probability of Model a being greater than 0.5. This criterion decreased the number of candidates to 23771 , including only 563 with $\mathrm{S} / \mathrm{N}>7$. Out of these 563 , the DMs of 401 candidates indicated that they are located beyond the Galaxy according to the YMW16 model. The visual inspection of these sorted candidates concluded that there are no potential FRB detections. We also used the score, which is the sum of probabilities determined from all classifier models (see Section 4) to sort all our candidates. The number of candidates with score $>3$ is 32254 , and 5102 of those have $\mathrm{S} / \mathrm{N}>7$. Only 920 candidates with $\mathrm{S} / \mathrm{N}>7$ have high enough DMs to place them outside our Galaxy, and the visual inspection determined that there is no potential FRB detection. The $\mathrm{S} / \mathrm{N}$ distribution of our candidates is shown in Fig. 6 for different score values and probabilities of Model a thresholds. We also noticed that strong RFI signals on March 28 produced many high $\mathrm{S} / \mathrm{N}$ single-pulse candidates that have DMs below $20 \mathrm{pc} \mathrm{cm}^{-3}$ in the search results. The classification models in FETCH, however, did not identify them as RFI and provided high probabilities. We discarded them only after visual inspection. We also ignored the candidates that have the same DM and are appeared simultaneously in multiple beams as they are highly like to be due to non-astrophysical signals. We also sorted out all candidates with Galactic DMs based on the probabilities of Model $a$ and also the score (see Fig. 6). We visually inspected these candidates, but none of them showed any evidence of being a credible pulse from a RRAT or a pulsar.

The classifier models evaluate the candidates mainly based on their training data sets and thus, there is a possibility that they can

19 http://www.naic. edu/ palfa/newpulsars

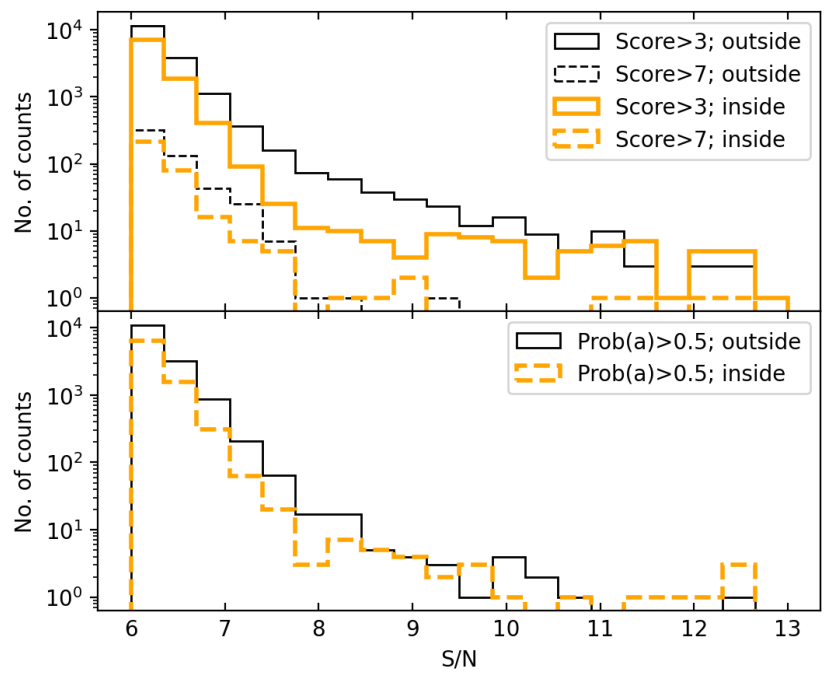

Figure 6. The $\mathrm{S} / \mathrm{N}$ distribution of single-pulse candidates that are placed outside (black) or inside (orange) the Galaxy using their DMs based on the electron density model YMW16. The top panel shows the distribution of candidates with score $>3$ (solid) and $>7$ (dashed). The bottom panel shows the distribution for candidates with probabilities $>0.5$ according to the classification Model $a$. All the candidates were visually inspected, but no potential FRBs or RRATs were identified.

produce false results (see Agarwal et al. 2020b). Therefore, we visually inspected candidates that have detection $\mathrm{S} / \mathrm{N}>7$ regardless of their classifier-model-produced probabilities, but we still did not find any evidence of a transient detection. We note there were a few candidates with a reasonably high $\mathrm{S} / \mathrm{N}$ detection; however, further examination indicated that these are mainly due to RFI or random noise. For example, Fig. 7(a) shows a FETCH-produced plot for a high $\mathrm{S} / \mathrm{N}(\approx 17)$, low DM candidate. By processing the data around the same candidate independently with the standard single-pulse routine (single_pulse_search.py) in PRESTO (Ransom 2011), we found that it is very likely due to RFI (see Fig. 7(b) and (c)). We also noticed a series of single pulses around the same candidate (with $\mathrm{DM}<70 \mathrm{pc} \mathrm{cm}^{-3}$ ) with slightly different time stamps. Therefore, we performed a periodicity search using PRESTO to test whether this emission is from a pulsar, but the analysis confirmed that this signal is local and due to RFI.

Furthermore, we searched for candidates that have the same sky location (within the beam width) and similar DM to identify repeaters. Such source can effectively identify due to its nature of repeating pulses and thus, we used a lower detection threshold of $\mathrm{S} / \mathrm{N}=6$ in the search. This analysis narrowed down a few sets of matching candidates, but visual inspection confirmed that they are likely due to noise and showed no evidence for detection of a repeater.

The major concern of candidate selection is how to distinguish real astrophysical signals and random noise when the candidate detection $\mathrm{S} / \mathrm{N}$ is low (e.g. $\lesssim 8$ ). Most of our candidates fall into this $\mathrm{S} / \mathrm{N}$ region (see Fig. 6). Yang et al. (2021) recently presented a sample of example FRB candidates with very low $\mathrm{S} / \mathrm{N}$ ratios using Parkes telescope archival data. The only way to prove that these weak events are astrophysical by re-detecting them in follow-up observations in the future. In order to understand the nature of candidates produced by random noise in our data, we searched for single-pulse candidates over 'negative' DM values, which are purely 'non-astrophysical' candidates produced by random noise. We selected for this purpose the $\sim 9 \mathrm{hr}$ data set of Beam0 on March 25, which produced 9003 can- 

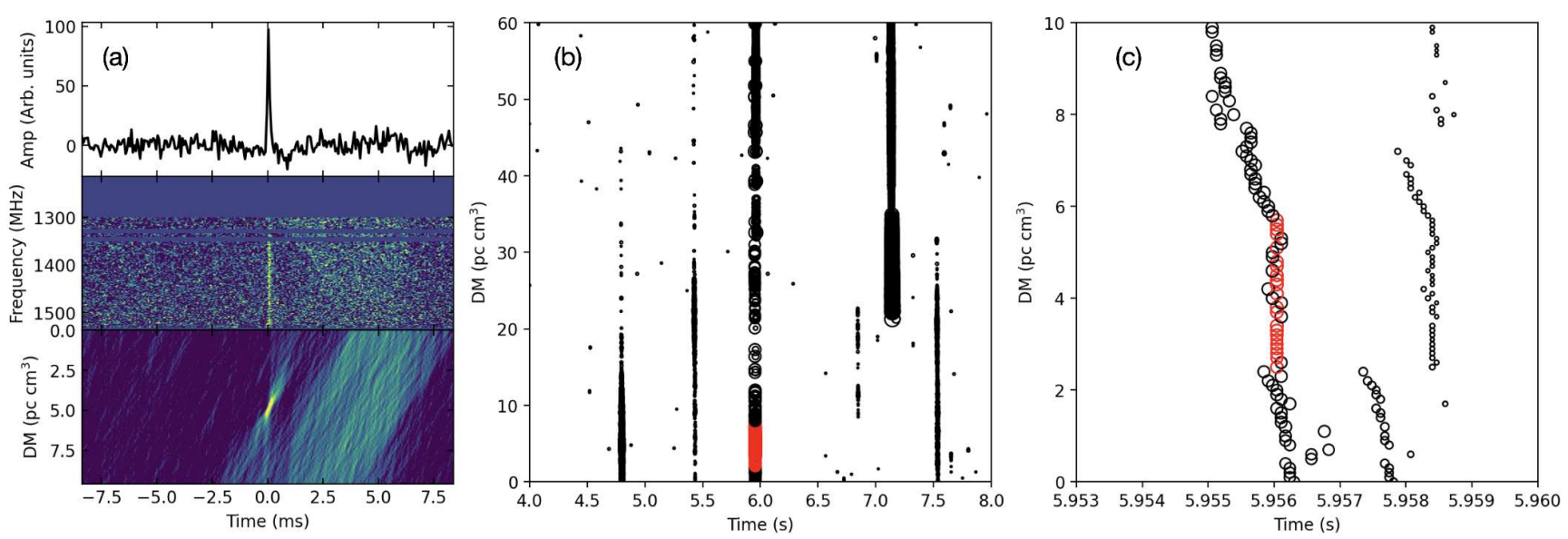

Figure 7. High $\mathrm{S} / \mathrm{N}(\approx 17)$ candidate (left panel) in Beam4 on March 22 with a low DM $\left(\approx 4.8 \mathrm{pc} \mathrm{cm}^{3}\right)$. The left-middle panel shows the candidate across the frequency band and the left-top panel shows the time series after averaging the signal across the frequency band. The left-bottom panel shows a color map of the pulse S/N across the DM-time space around its best reported DM value of $4.8 \mathrm{pc} \mathrm{cm}^{3}$. The candidate is independently validated using the single-pulse routine in the PRESTO package and the DM versus time results are shown in panel (b) and (c). The circles represent a single-pulse detection at that particular DM and time, and the radius of the circle is scaled according to the detected $\mathrm{S} / \mathrm{N}$. The candidate on panel (a) corresponds to a signal around the time of $\sim 5.9 \mathrm{~s}$ on panel (b), which is shown in red circles. Panel (c) shows a zoomed-in version of panel (b) around the same candidate, indicating that the signal is not well constrained in DM and the candidate is likely due to RFI.

didates with $\mathrm{S} / \mathrm{N}>6$ over the DM range of $1-11000 \mathrm{pc} \mathrm{cm}^{-3}$ in our standard search (described in Section 4). We fed the same data set into our pipeline with the same DM range, but for negative values (i.e., between -11000 to $-1 \mathrm{pc} \mathrm{cm}^{-3}$ ). This analysis produced 10525 purely non-astrophysical candidates with $\mathrm{S} / \mathrm{N}>6$, which is more than the number of candidates found in the standard search. We also note that these non-astrophysical, random noise candidates are visually very similar to low $\mathrm{S} / \mathrm{N}$ candidates in our standard search. This test suggests that low $\mathrm{S} / \mathrm{N}$ candidates are not easily distinguished from real astrophysical signals and random noise. Thus, we conclude that the low $\mathrm{S} / \mathrm{N}$ candidates in the distribution are due to random noise.

\section{KNOWN PULSARS AND TRANSIENTS}

As shown in Fig. 3, there are five known pulsars that have positions covered by our observations. However, the single pulses of these sources were not detected through our pipeline. Perhaps these pulses were too weak to be detected with the noise level and our detection thresholds. In this section, we searched for their emission by folding the data using their timing ephemerides. The basic properties of these sources are given in Table 1. PSR J1627+1419 was within our observations on March 19, and it was detected in Beam1 with an offset of $0.5^{\prime}$ from the beam centre. This slow pulsar has a period of $0.491 \mathrm{~s}$ (Foster et al. 1995) and it is bright at frequencies $<800 \mathrm{MHz}$ with fluxes of 78, 6, and $4 \mathrm{mJy}$ at 150,430 , and $774 \mathrm{MHz}$, respectively, resulting in a spectral index of $-1.6 \pm 0.3$ (Bilous et al. 2016; Lewandowski et al. 2004; Han et al. 2009). We extracted the $\sim 13$ s data chunk in which the pulsar crossed Beam1 during its transit. We then processed this data using the ephemeris of the pulsar (Foster et al. 1995) via the pulsar signal processing package DSPSR (van Straten \& Bailes 2011). The pulsar emission is seen in the processed data, and the integrated pulse profile is shown in Fig. 8 (see left panel). We then estimated the flux density of the pulsar using the radiometer noise given in Equation A1. Multiplying this equation by $1 / G$, where $G$ is the telescope gain, we can estimate the noise fluctuation in Jy (see Equation 7.12 in Lorimer \& Kramer 2012). Assuming $\Delta f=215 \mathrm{MHz}, t_{\mathrm{obs}}=13 \mathrm{~s}$, and $G=7.1 \mathrm{~K} \mathrm{Jy}^{-1}$ (see
Section 2.2), as well as other standard values given in Appendix A, we scaled the amplitude of the pulse profile in mJy (see Fig. 8) and then estimated the mean flux density. The mean flux density we obtained is $0.6 \pm 0.1 \mathrm{mJy}$, which is within the errors of the expected mean flux density of $0.9 \pm 0.4 \mathrm{mJy}$ at $1.4 \mathrm{GHz}$ based on the spectral index of $-1.6 \pm 0.3$ (Bilous et al. 2016).

PSR J0625+10 happened to be within Beam3 on January 27 ( $1.4^{\prime}$ off from the beam center). This pulsar has a spin period of $\sim 0.5 \mathrm{~s}, \mathrm{DM}$ of $78 \mathrm{pc} \mathrm{cm}^{-3}$ (Camilo et al. 1996), and a very low flux density of $0.09 \mathrm{mJy}$ at $1.4 \mathrm{GHz}$ (Lazarus et al. 2015); see Table 1. We again selected a $\sim 13 \mathrm{~s}$ data section during which the pulsar was within Beam 3 and processed it using the ephemeris of the pulsar. The processed data is shown in Fig. 8 (right panel), and the observed pulse profile has a $\mathrm{S} / \mathrm{N}$ of 8 . The mean flux density is estimated to be $0.03 \pm 0.10 \mathrm{mJy}$, following the same method given above, which is within the errors of the previously reported value (see Table 8 and also Lazarus et al. 2015).

We also noticed that PSR J1329+13 was within Beam0 (offset by $0.5^{\prime}$ from the beam centre) during the March 17 session based on the pulsar position reported in Tyul'bashev et al. (2018). This is a RRAT with a DM of $12 \pm 2 \mathrm{pc} \mathrm{cm}^{-3}$, and its spin period is currently unknown. To identify emitted single pulses from the pulsar in our single-pulse analysis (see Section 4), we searched over all our candidates to find those that matched the DM and position of the pulsar. We used a tolerance of $\pm 2 \mathrm{pc} \mathrm{cm}^{-3}$ in DM to narrow down any possible candidates and constrained the position to be within our beam size. However, we did not find any convincing candidates that match the pulsar DM and position. PSR J1329+13 was discovered at a frequency of $111 \mathrm{MHz}$ with a high flux density (Tyul'bashev et al. 2018), suggesting that it should be detected in our data. That we did not detect this pulsar could imply that it was in the emission off state when it transited the beam. We further note that the current position of this pulsar is poorly constrained, RA $=13: 29$ (2) and Dec $=+13: 44$ (20) (Tyul'bashev et al. 2018), in which case perhaps it was never actually within the beam.

PSR J1749+16 is a $2.3 \mathrm{~s}$ pulsar with a DM of $59.6 \mathrm{pc} \mathrm{cm}^{-3}$ (Deneva et al. 2016); see Table 1. It was in Beam6 on March 23, 
Table 1. The properties of known pulsars and FRBs that have positions covered by our observations. The previously reported mean flux densities $S$ at 1.4 GHz and fluences at $600 \mathrm{MHz}$ of these pulsars and FRBs are given in sixth and seventh columns, respectively. The last column noted the detectability of these sources in our data. Note that none of these FRBs have been identified as repeaters.

\begin{tabular}{lccccccc}
\hline \multicolumn{1}{c}{ Source } & $\begin{array}{c}\text { Period } \\
(\mathrm{ms})\end{array}$ & $\begin{array}{c}\mathrm{DM} \\
\left(\mathrm{pc} \mathrm{cm}^{-3}\right)\end{array}$ & $\begin{array}{c}l \\
\left({ }^{\circ}\right)\end{array}$ & $\begin{array}{c}b \\
\left({ }^{\circ}\right)\end{array}$ & $\begin{array}{c}S \\
(\mathrm{mJy})\end{array}$ & $\begin{array}{c}\text { Fluence } \\
(\mathrm{Jy} \mathrm{ms})\end{array}$ & Detected \\
\hline PSR J0625+10 & 498 & 78 & 200.88 & -0.96 & 0.09 & - & Yes \\
PSR J1329+13 & - & 12 & 338 & 73.99 & - & - & No \\
PSR J1627+1419 & 491 & 32.2 & 30.03 & 38.32 & $0.95^{\dagger}$ & - & Yes \\
PSR J1651+14 & 828 & 48 & 32.88 & 33.07 & - & - & No \\
PSR J1749+16 & 2312 & 59.6 & 41.21 & 20.90 & - & - & No \\
\hline FRB20180906A & - & 383.46 & 217.17 & 40.61 & - & 3 & No \\
FRB20190131C & - & 507.76 & 241.74 & 60.05 & - & 2 & No \\
FRB20190214C & - & 533.11 & 20.45 & 64.93 & - & 5 & No \\
FRB20190224C & - & 497.4 & 203.48 & 27.2 & - & 8 & No \\
\hline
\end{tabular}

${ }^{\dagger}$ The expected flux density of the pulsar estimated at $1.4 \mathrm{GHz}$ based on its measured spectral index of -1.6 given in Bilous et al. (2016).
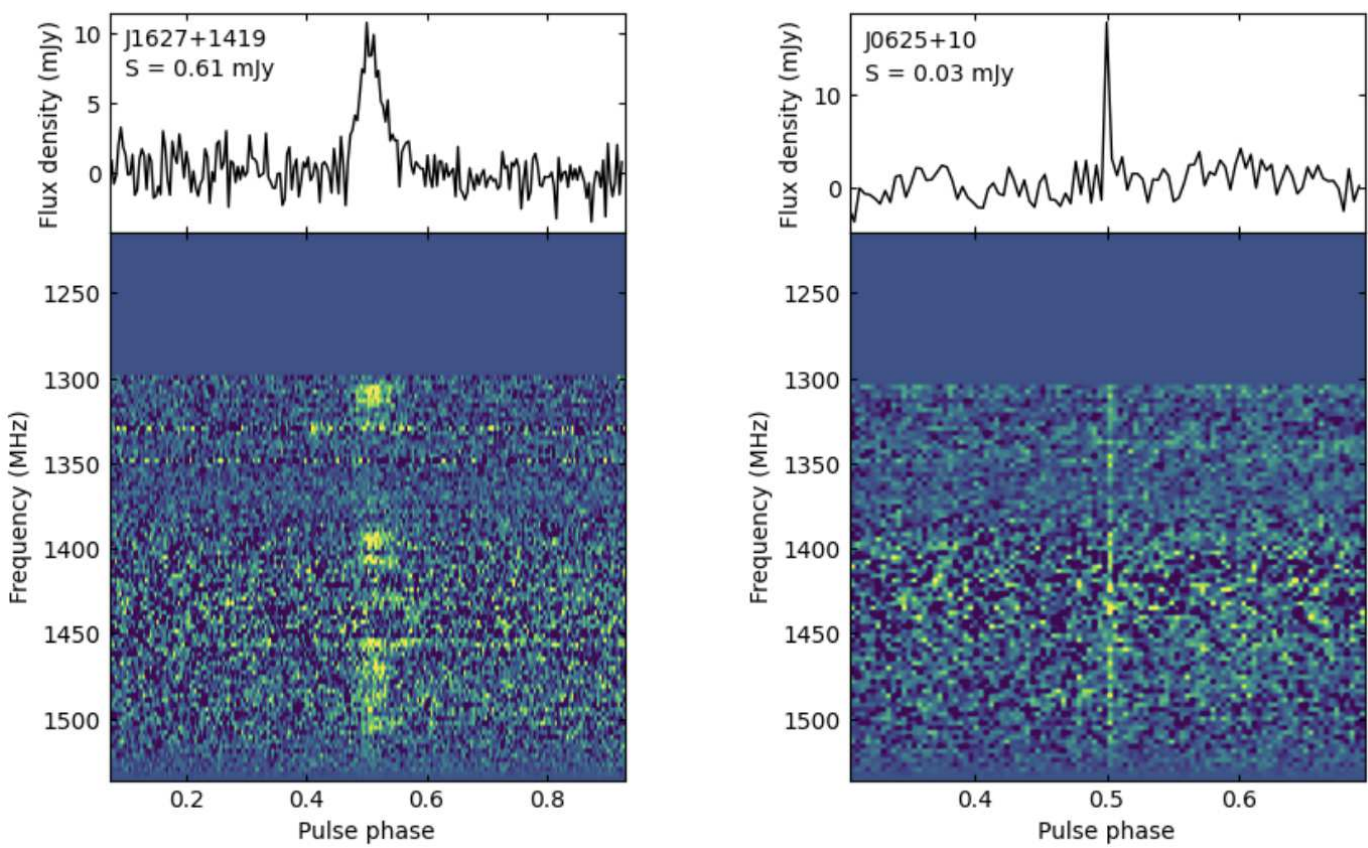

Figure 8. Detected emission from known pulsars by folding the data using their timing ephemerides. PSR J1627+1419 was within Beam1 (with an offset of $0.5^{\prime}$ from the beam center) on March 19, and J0625+10 was within Beam3 (with an offset of 1.4' from the beam center) on January 27. The top panel shows the integrated pulse profile, folded over $13 \mathrm{~s}$ of data, and the bottom panel shows the emission across the frequency band. The mean flux densities for J1627+1419 and J0625+10 are estimated to be $0.6 \pm 0.1$ and $0.03 \pm 0.1 \mathrm{mJy}$, respectively. For clarity, only a portion of the pulse phase is shown.

and while we applied the same procedure as described above, we did not detect emission from the pulsar. We note that J1749+16 is a nulling pulsar with nulls for tens of seconds, suggesting that perhaps it was in the null state when it transited across the beam, resulting in non-detection.

PSR J1651+14 is a $0.83 \mathrm{~s}$ pulsar with a DM of $48 \mathrm{pc} \mathrm{cm}^{-3}$ (Tyul'bashev et al. 2017), and it was in Beam6 on March 19. As for other pulsars, we cropped the data section based on the reported position of the pulsar given in Tyul'bashev et al. (2017) and then processed the data; however, we did not detect pulsar emission. We note that the discovery of the pulsar was very weak and carried out at $111 \mathrm{MHz}$. No other observations have been reported for this pulsar. Perhaps the pulsar is intrinsically weak and has a steep spectrum, so that its emission may not be visible at $1.4 \mathrm{GHz}$ with our $13 \mathrm{~s}$ integration time.

The FRBs given in Table 1 and also shown in Fig. 3 also happened to be within the ALFA beams. In order to check for repeating emission from these FRBs, we selected the single-pulse candidates produced when these sources were within the beams and then searched it for candidates with DMs similar to these sources. However, we could not find any repeating emission from these FRBs in our analysis. We also note that these sources have not been reported as repeaters (see The CHIME/FRB Collaboration et al. 2021) and thus it is unlikely to expect detection of these sources in our data.

Finally, magnetars can also produce FRB-like fast transient signals (see CHIME/FRB Collaboration et al. 2020; Bochenek et al. 
2020; Bailes et al. 2021). Therefore, we looked for magnetars ${ }^{20}$ that were within our observations based on their sky positions (Olausen \& Kaspi 2014; Palliyaguru et al. 2021) in order to search for transient emission from them. However, none of these sources were within our beams.

\section{DISCUSSION}

We collected 160 hrs of drift-scan data over 23 days in January and March 2020 with the Arecibo telescope. We processed the data and searched for fast transients, FRBs and RRATs, using a singlepulse pipeline that includes the HEIMDALL and FETCH packages. The pipeline produced over $8 \times 10^{6}$ single-pulse candidates, and the neural networks classification models in FETCH reduced this number to $\sim 24000$ "good" candidates with probabilities $>0.5$. Out of the remaining candidates, only $<1000$ had $\mathrm{S} / \mathrm{N}>7$ (see Section 5). We proceeded to inspect these candidates manually, but we did not identify any transient detections. We also searched over all of the candidates to find repeating transients by matching their DMs and sky locations, but again we found no evidence of detection for these sources. While there were no transient detections, we did observe emission from two known pulsars (PSRs J1627+1419 and J0625+10), and their measured flux densities are consistent with the expected values (see Fig. 8 and Section 6).

We finally estimated limits on the FRB event rates based on our observations, and compared that with other published rate estimates. Given a beam FoV of $0.022 \mathrm{deg}^{2}$ and $\sim 160 \mathrm{hrs}$ of observations in our campaign, we estimate the upper limit of the FRB event rate as $<2.8 \times 10^{5} \mathrm{sky}^{-1} \mathrm{~d}^{-1}$ at $1.4 \mathrm{GHz}$ above a fluence of $0.16 \mathrm{Jy} \mathrm{ms}$. We also estimated the FRB rate using the published rates based on the detections from other telescopes. Since the Parkes telescope detected FRBs at $1.4 \mathrm{GHz}$ and our observations were also at the same frequency band, we used the Parkes FRB rate to estimate the expected FRB rate for our observations. Thornton et al. (2013) reported FRB detections using Parkes and estimated the rate as $10000 \mathrm{sky}^{-1} \mathrm{~d}^{-1}$. Keane \& Petroff (2015) reanalyzed Thornton et al. (2013) results and derived a fluence complete event rate of $2500 \mathrm{sky}^{-1} \mathrm{~d}^{-1}$ above a fluence of $2 \mathrm{Jy} \mathrm{ms}$. As described in Section 3, the sensitivity of our observations is estimated at a fluence of $0.16 \mathrm{Jy} \mathrm{ms}$ assuming a pulse width of $5 \mathrm{~ms}$. In order to convert the sensitivity of Parkes to Arecibo, we use the FRB power-law flux distribution $N(>F) \propto F^{-\gamma}$, assuming a Euclidean Universe with $\gamma=3 / 2$, where the sources are assumed to be non-evolving and uniformly distributed in space (Connor et al. 2016). We also note that $\gamma$ is constrained using the FRBs detected with the CHIME telescope to be 1.4 (The CHIME/FRB Collaboration et al. 2021), which is an excellent match to an Euclidean space source distribution. Using this flux distribution for the Parkes event rate with its own fluence given above, we scaled the FRB rate to $1.1 \times 10^{5} \mathrm{sky}^{-1} \mathrm{~d}^{-1}$ above our fluence limit of $0.16 \mathrm{Jy}$ ms. The recent FRB detections reported by CHIME constrained the event rate to be $818 \mathrm{sky}^{-1} \mathrm{~d}^{-1}$ above a fluence of $5 \mathrm{Jy} \mathrm{ms}$ at $600 \mathrm{MHz}$ (The CHIME/FRB Collaboration et al. 2021). Using the above mentioned flux distribution with a flat spectral index, we then scaled the event rate to be $1.4 \times 10^{5} \mathrm{sky}^{-1} \mathrm{~d}^{-1}$ above our fluence limit of $0.16 \mathrm{Jy} \mathrm{ms}$ at $1.4 \mathrm{GHz}$. The spectral properties of FRBs are still poorly understood and thus, a flat distribution is a valid assumption (see Macquart et al. 2019; Farah et al. 2019; The CHIME/FRB Collaboration et al. 2021, for discussion).

20 http://www.physics.mcgill.ca/ pulsar/magnetar/main.html
Finally, we estimated the average observing time required for an Arecibo-like telescope to detect at least one FRB using the event rate of $1.1 \times 10^{5} \mathrm{sky}^{-1} \mathrm{~d}^{-1}$ estimated above. Given the ALFA beam FoV of $0.022 \mathrm{deg}^{2}$, the average required observation time to detect one FRB is approximately 410 hours. Assuming eight hours of continuous observations every day, we need, on average, at least 51 days of observations, which is a factor of 2.2 longer than we spent in our campaign. Note that we conducted these observations during the observatory closure to fill the downtime of the telescope, so that we could not observe more than 160 hours to satisfy the above detection requirement.

Currently, we are processing our data to search for pulsars using periodicity searches. Even though the sky transit time within our beams is $\sim 13 \mathrm{~s}$, we emphasize that it is worth searching for pulsars in our data, and bright pulsars, especially those with short periods, may appear with a reasonable $\mathrm{S} / \mathrm{N}$ ratio (see the known pulsar detections in our data that described in Section 8). In parallel to the periodicity search, we have included PRESTO-based (Ransom et al. 2002; Keith et al. 2011; Ransom 2011) single-pulse routines (single_pulse_search.py ${ }^{21}$ ) in our pipeline to conduct an independent transients search analysis. The data processing is underway, and we will relay those results in a future publication.

We conclude by noting that our transient search pipeline is currently under modification to perform real-time searches, and it will be tested on the 12-m telescope at the Arecibo Observatory. This telescope is undergoing an upgrade to integrate a cooled receiver system $^{22,23}$. A significant portion of the telescope time will be dedicated to real-time transient searches, along with commensal observations, in the future.

\section{DATA AVAILABILITY}

The data underlying this article will be shared on reasonable request to the corresponding author.

\section{ACKNOWLEDGMENTS}

The Arecibo Observatory is operated by the University of Central Florida, Ana G. Mendez-Universidad Metropolitana, and Yang Enterprises under a cooperative agreement with the National Science Foundation (NSF; AST-1744119). We thank Emilie Parent for useful discussions about some of the data processing tools.

\section{REFERENCES}

Agarwal D., Aggarwal K., 2020, devanshkv/fetch: Software release with the manuscript, doi:10.5281/zenodo.3905437, https://doi.org/10.5281/zenodo. 3905437

Agarwal D., et al., 2020a, MNRAS, 497, 352

Agarwal D., Aggarwal K., Burke-Spolaor S., Lorimer D. R., Garver-Daniels N., 2020b, MNRAS, 497, 1661

Aggarwal K., Agarwal D., Lewis E. F., Anna-Thomas R., Cardinal Tremblay J., Burke-Spolaor S., McLaughlin M. A., Lorimer D. R., 2021, arXiv e-prints, p. arXiv:2107.05658

Bailes M., et al., 2021, MNRAS, 503, 5367

Bannister K. W., et al., 2017, ApJ, 841, L12

21 https://github.com/scottransom/presto

22 http://www.naic.edu/ phil/hardware/12meter/patriot12meter.html

23 http://www.naic.edu/ao/scientist-user-portal/astronomy/under-devel 
Bannister K. W., et al., 2019, Science, 365, 565

Barsdell B. R., Bailes M., Barnes D. G., Fluke C. J., 2012, MNRAS, 422, 379

Beniamini P., Wadiasingh Z., Metzger B. D., 2020, MNRAS, 496, 3390

Bethapudi S., Desai S., 2018, Astronomy and Computing, 23, 15

Bhandari S., et al., 2021, arXiv e-prints, p. arXiv:2108.01282

Bilous A. V., et al., 2016, A\&A, 591, A134

Bochenek C. D., Ravi V., Belov K. V., Hallinan G., Kocz J., Kulkarni S. R., McKenna D. L., 2020, Nature, 587, 59

CHIME/FRB Collaboration et al., 2018, ApJ, 863, 48

CHIME/FRB Collaboration et al., 2019a, Nature, 566, 230

CHIME/FRB Collaboration et al., 2019b, Nature, 566, 235

CHIME/FRB Collaboration et al., 2019c, ApJ, 885, L24

CHIME/FRB Collaboration et al., 2020, Nature, 587, 54

Caleb M., et al., 2017, MNRAS, 468, 3746

Camilo F., Nice D. J., Shrauner J. A., Taylor J. H., 1996, ApJ, 469, 819

Chatterjee S., et al., 2017, Nature, 541, 58

Chime/Frb Collaboration et al., 2020, Nature, 582, 351

Choudhury T. R., Padmanabhan T., 2005, A\&A, 429, 807

Condon J. J., Cotton W. D., Greisen E. W., Yin Q. F., Perley R. A., Taylor G. B., Broderick J. J., 1998, AJ, 115, 1693

Connor L., van Leeuwen J., 2018, AJ, 156, 256

Connor L., Lin H.-H., Masui K., Oppermann N., Pen U.-L., Peterson J. B., Roman A., Sievers J., 2016, MNRAS, 460, 1054

Connor L., et al., 2020, MNRAS, 499, 4716

Cordes J. M., McLaughlin M. A., 2003, ApJ, 596, 1142

Cordes J. M., et al., 2006, ApJ, 637, 446

Cruces M., et al., 2021, MNRAS, 500, 448

Deneva J. S., et al., 2009, ApJ, 703, 2259

Deneva J. S., et al., 2016, ApJ, 821, 10

Devine T. R., Goseva-Popstojanova K., McLaughlin M., 2016, MNRAS, 459,1519

Farah W., et al., 2019, MNRAS, 488, 2989

Foster R. S., Cadwell B. J., Wolszczan A., Anderson S. B., 1995, ApJ, 454, 826

Foster G., et al., 2018, MNRAS, 474, 3847

Gajjar V., et al., 2018, ApJ, 863, 2

Guo P., Duan F., Wang P., Yao Y., Yin Q., Xin X., 2017, arXiv e-prints, p. arXiv:1711.10339

Gupta V., et al., 2021, MNRAS, 501, 2316

Han J. L., Demorest P. B., van Straten W., Lyne A. G., 2009, ApJS, 181, 557

Heintz K. E., et al., 2020, ApJ, 903, 152

Ioka K., 2003, ApJ, 598, L79

Ioka K., Zhang B., 2020, ApJ, 893, L26

Karastergiou A., et al., 2015, MNRAS, 452, 1254

Keane E. F., Petroff E., 2015, MNRAS, 447, 2852

Keating L. C., Haehnelt M. G., Cantalupo S., Puchwein E., 2015, MNRAS, 454,681

Keith M. J., et al., 2011, MNRAS, 414, 1292

Komatsu E., et al., 2009, ApJS, 180, 330

Kumar P., et al., 2019, ApJ, 887, L30

Law C. J., et al., 2020, ApJ, 899, 161

Lazarus P., et al., 2015, ApJ, 812, 81

Levin Y., Beloborodov A. M., Bransgrove A., 2020, ApJ, 895, L30

Lewandowski W., Wolszczan A., Feiler G., Konacki M., Sołtysiński T., 2004, ApJ, 600, 905

Lorimer D. R., 2018, Nature Astronomy, 2, 860

Lorimer D. R., Kramer M., 2012, Handbook of Pulsar Astronomy

Lorimer D. R., Bailes M., McLaughlin M. A., Narkevic D. J., Crawford F., 2007, Science, 318, 777

Luo R., et al., 2020, Nature, 586, 693

Lyutikov M., Popov S., 2020, arXiv e-prints, p. arXiv:2005.05093

Lyutikov M., Barkov M. V., Giannios D., 2020, ApJ, 893, L39

Macquart J. P., Shannon R. M., Bannister K. W., James C. W., Ekers R. D., Bunton J. D., 2019, ApJ, 872, L19

Macquart J. P., et al., 2020, Nature, 581, 391

Marcote B., et al., 2020, Nature, 577, 190

Masui K., et al., 2015, Nature, 528, 523

McLaughlin M. A., et al., 2006, Nature, 439, 817
Michilli D., et al., 2018, Nature, 553, 182

Niu C.-H., et al., 2021, ApJ, 909, L8

Olausen S. A., Kaspi V. M., 2014, ApJS, 212, 6

Palliyaguru N. T., Agarwal D., Golpayegani G., Lynch R., Lorimer D. R., Nguyen B., Corsi A., Burke-Spolaor S., 2021, MNRAS, 501, 541

Parent E., et al., 2020, ApJ, 904, 92

Parent E., et al., 2021, arXiv e-prints, p. arXiv:2108.02320

Patel C., et al., 2018, ApJ, 869, 181

Piro L., et al., 2021, arXiv e-prints, p. arXiv:2107.14339

Prochaska J. X., et al., 2019, Science, 366, 231

Rajwade K. M., et al., 2020, MNRAS, 495, 3551

Ransom S., 2011, PRESTO: PulsaR Exploration and Search TOolkit (ascl:1107.017)

Ransom S. M., Eikenberry S. S., Middleditch J., 2002, AJ, 124, 1788

Ravi V., et al., 2019, Nature, 572, 352

Shannon R. M., et al., 2018, Nature, 562, 386

Sob'yanin D. N., 2020, MNRAS, 497, 1001

Spitler L. G., et al., 2014, ApJ, 790, 101

Spitler L. G., et al., 2016, Nature, 531, 202

Surnis M. P., et al., 2019, Publ. Astron. Soc. Australia, 36, e032

The CHIME/FRB Collaboration et al., 2021, arXiv e-prints, p. arXiv:2106.04352

Thornton D., et al., 2013, Science, 341, 53

Tyul'bashev S. A., et al., 2017, Astronomy Reports, 61, 848

Tyul'bashev S. A., et al., 2018, Astronomy Reports, 62, 63

Wagstaff K. L., et al., 2016, PASP, 128, 084503

Wang F. Y., Wang Y. Y., Yang Y.-P., Yu Y. W., Zuo Z. Y., Dai Z. G., 2020, ApJ, 891, 72

Xiao D., Wang F., Dai Z., 2021, Science China Physics, Mechanics, and Astronomy, 64, 249501

Yang H., Zou Y.-C., 2020, ApJ, 893, L31

Yang X., et al., 2021, arXiv e-prints, p. arXiv:2108.00609

Yao J. M., Manchester R. N., Wang N., 2017, ApJ, 835, 29

Zhang B., 2018, ApJ, 867, L21

Zhang B., 2020a, Nature, 587, 45

Zhang B., 2020b, ApJ, 890, L24

Zhang Y. G., Gajjar V., Foster G., Siemion A., Cordes J., Law C., Wang Y., 2018, ApJ, 866, 149

Zhu W. W., et al., 2014, ApJ, 781, 117

Zhu W., et al., 2020, ApJ, 895, L6

van Straten W., Bailes M., 2011, Publ. Astron. Soc. Australia, 28, 1

\section{APPENDIX A: TELESCOPE GAIN AND STABILITY ESTIMATION}

The telescope gain was obtained using the time series corresponding to a spectral channel from the observations during the transit of a continuum source. The spectral channel was selected in a way such that the data is not affected by RFI. Since the expected source transit time within our beams is $\sim 13 \mathrm{~s}$, we average the raw data to make $1 \mathrm{~s}$ integration intervals. The NVSS source catalog was used to identify the sources and obtain their flux densities (Condon et al. 1998). The sources with flux densities greater than $100 \mathrm{mJy}$ at $1.4 \mathrm{GHz}$ and those fall within $1^{\prime}$ of the beam were selected. This criteria resulted in at least one source in each beam almost everyday. This allows us to estimate the gain every day for each beam, determining the stability of the system. The gains were estimated using two methods.

The first method uses the radiometer equation, where the expected root mean square noise fluctuation

$\Delta T_{\text {sys }}=\frac{T_{\text {sys }}}{\sqrt{n_{\mathrm{p}} t_{\text {obs }} \Delta f}}=C \sigma_{\mathrm{p}}$

where $T_{\text {sys }}$ is the system temperature, $n_{\mathrm{p}}$ is the number of polarization channels summed, $t_{\mathrm{obs}}$ is integration time, $\Delta f$ is observing 
bandwidth, and $\sigma_{\mathrm{p}}$ is the standard deviation of the off-source region in the time series. The proportionality constant $C$ is in units of $\mathrm{K}$ per count and used to scale the ordinate of the time series. In the calculation, we used the typical value $T_{\text {sys }}=28 \mathrm{~K}$ of ALFA receiver, $\Delta f=0.335 \mathrm{MHz}, n_{\mathrm{p}}=2$, and $t_{\mathrm{obs}}=1 \mathrm{~s}$. The constant $C$ is then used to convert the on-source deflection in counts to units of $\mathrm{K}$. The telescope gain $G$ is the ratio of the deflection in units of $\mathrm{K}$ to the flux density of the source in Jy. The direct estimate of $\sigma_{\mathrm{p}}$ from the time series may affect by the gain variation over time and confusion noise. Therefore, we computed $\sigma_{\mathrm{p}}$ value from the difference between the time series from adjacent channels, leading to cancel out both these effects.

In the second method, we estimated the gain using the median of the off-source region of the time series, which is proportional to $T_{\text {sys }}$. Thus, the ratio of $T_{\text {sys }}$ to median value gives the conversion factor in units of K per count. The on-source deflection can then be converted to $\mathrm{K}$ using this factor and the telescope gain can be estimated as described above.

The gain estimated using these two methods are found to be comparable with each other. Therefore, we averaged the gain obtained from the two methods for each beam. We note that one of the polarization channels of Beam5 was unstable and poorly behaved, so that it was ignored in the gain estimation.

This paper has been typeset from a $\mathrm{T}_{\mathrm{E}} \mathrm{X} / \mathrm{L} \mathrm{T}_{\mathrm{E}} \mathrm{X}$ file prepared by the author. 